\title{
Autoimmune Aspects of Type 2 Diabetes Mellitus - A Mini-Review
}

\author{
Bianca K. Itariu ${ }^{a, b}$ Thomas M. Stulnig ${ }^{a, b}$ \\ ${ }^{a}$ Christian Doppler Laboratory for Cardio-Metabolic Immunotherapy and ${ }^{\mathrm{b}}$ Clinical Division of Endocrinology and \\ Metabolism, Department of Medicine III, Medical University of Vienna, Vienna, Austria
}

\section{Key Words}

Autoimmunity · Autoantibodies · Type 2 diabetes .

Inflammation $\cdot T$ cells $\cdot B$ cells

\begin{abstract}
Autoimmunity is a well-known pathogenic component in type 1 diabetes (T1DM). The assumption that the pathogenesis of type 2 diabetes (T2DM) also encompasses autoimmune aspects is recognized increasingly, based on the presence of circulating autoantibodies against $\beta$ cells, self-reactive T cells, but also on the glucose-lowering efficacy of some immunomodulatory therapies in T2DM. The identification of these autoantibodies in elderly patients with slowly progressive manifestation of diabetes led to the introduction of a distinct clinical entity termed latent autoimmune diabetes of the adult (LADA), which combines features of both T1DM and T2DM. The autoantibody cluster differs in patients with LADA from patients with T1DM, but their presence indicates steady progression towards $\beta$-cell death and subsequent need for initiation of insulin treatment in a shorter period of time compared to autoantibody-negative T2DM patients. Autoimmune aspects in T2DM are not solely restricted to autoantibodies and thus LADA. They include the self-reactive $T$ cells or defects in regulatory $T$ cells (Tregs), which have been detected in autoantibody-negative T2DM patients as well. One contributor to the autoimmune activation in T2DM seems to be the chronic inflammatory state, characteristic of this disease. Upon inflammation-induced tissue destruction, cryptic 'self' antigens can trigger an autoimmune response,
\end{abstract}

which in turn accelerates $\beta$-cell death. Both innate and adaptive immune system components, specifically macrophages and self-reactive $T$ cells, contribute to an increased secretion of inflammatory cytokines involved in inflammatory and autoimmune processes. However, the extent to which inflammation overlaps with autoimmunity is not known. Our review focuses on autoimmune involvement in T2DM, with an emphasis on LADA and the humoral immune response, on the involvement of chronic inflammation in autoimmunity, and specifically the role of $B$ and $T$ cells as links between inflammatory and autoimmune reactions. We will further stress the consequences of autoimmune activation for T2DM patients and present novel therapeutic approaches for T2DM management that rely on immune modulation.

(C) 2014 S. Karger AG, Basel

\section{Introduction}

Autoimmunity is a highly complex, multifactorial process defined by loss of self-tolerance and chronic excess reactivity of $\mathrm{B}$ and $\mathrm{T}$ cells stemming danger signals released when cells or tissues undergo abnormal cell death $[1,2]$. Multiple factors are involved in its pathogenesis, from genetics to environment [3]. Indicators of autoimmune involvement include the persistent presence of circulating autoantibodies or self-reactive $\mathrm{T}$ cells which affect target organs, evidence of lymphocytic infiltrates in target organs, tissue damage, association with other autoimmune disease, and evidence of beneficial effects from

\section{KARGER}

E-Mail karger@karger.com

www.karger.com/ger (c) 2014 S. Karger AG, Basel

0304-324X/14/0603-0189\$39.50/0
Prof. T.M. Stulnig, MD

Christian Doppler Laboratory for Cardio-Metabolic Immunotherapy

Clinical Division of Endocrinology and Metabolism, Department of Medicine III

Medical University of Vienna, Währinger Gürtel 18-20, AT-1090 Vienna (Austria)

E-Mail thomas.stulnig@meduniwien.ac.at 
immunosuppressive therapy. Manifestations of autoimmunity are detectable in numerous pathologic conditions, which do not fall under the category of autoimmune diseases. Type 2 diabetes (T2DM) is one such example. Sustained research over the last 30 years has challenged the stereotypical view that T2DM is solely a metabolic disease by identifying autoimmunity as an overlapping feature of type 1 diabetes (T1DM) and T2DM, which leads to impaired insulin secretion in $\beta$ cells and promotes hyperglycaemia [4].

Diabetes development based on combined cellular autoimmunity and insulin resistance has been reflected by various terms, such as double diabetes, latent autoimmune diabetes of the adult (LADA) or the young (LADY) or type 1.5 diabetes mellitus. Its nosographic characterization is yet a matter of debate, thus many T2DM patients may go undiagnosed for autoimmune $\beta$-cell alterations, which may have therapeutic consequences. LADA patients, who are generally defined by age of diagnosis $>30$ years, presence of circulating islet autoantibodies, and lack of insulin requirement for 6 months after diagnosis [5], need insulin earlier during disease progression, are likely to respond poorly to oral antidiabetic mediation [6], but they could respond favourably to immunomodulator therapy. However, anti-inflammatory and immunomodulatory therapies have also proven effective in improving the metabolic profile of many T2DM patients, possibly by interfering with autoimmune processes and thereby halting the decline of $\beta$-cell function [7].

Obesity is by far the main underlying factor causing T2DM and its pathological potential lies in obesity-associated insulin resistance, activation of innate immunity and chronic low-grade inflammation [7]. The chronic inflammatory state in T2DM is characterized by an increased production of cytokines, most notably interleukin (IL)- $1 \beta$, which destroy $\beta$ cells [7]. When tissue inflammation-induced tissue destruction occurs, 'self antigens, which are generally not accessible to T cells, can be released from the affected tissues and thus promote autoimmune activation [1]. Concomitantly, many more alarm signals such as various cytokines or extracellular matrix breakdown products can be released into the circulation if cellular death occurs by dysfunctional apoptosis [8]. For approaching autoimmunity in the context of T2DM, one also has to take into account the role of apoptosisrelated molecules Fas and Fas ligand and defects in active suppression of self-reactivity by regulatory T cells (Tregs), immune deviations in the T-helper 1/T-helper 2 (Th1/ Th2) ratio or defects in B-cell tolerance [9-11].
Our review focuses on existing evidence on autoimmune aspects of T2DM from a clinical, pathophysiological and therapeutic perspective. We will discuss (i) clinical traits and therapeutic implications of LADA patients, since they distinctively illustrate how autoimmunity connects the spectrum of T1DM and T2DM, (ii) possible causes of autoimmune activation in T2DM, with an emphasis on the immunogenic potential of chronic lowgrade inflammation as continuous supply of danger signals which activate pattern recognition receptors, and (iii) anti-inflammatory and immunomodulating therapies with anti-diabetic effects as well as anti-diabetic medication affecting immune regulation.

\section{LADA at the Crossroad between T1DM and T2DM}

A hallmark of autoimmune involvement in T2DM is the presence of circulating autoantibodies. Consistent evidence of islet cell autoimmunity in T2DM patients was reported in 1997 by Turner et al. [12], with the identification of glutamic acid decarboxylase (GAD) and islet cytoplasm autoantibodies in $12 \%$ of over 3,000 T2DM patients aged between 25 and 65 years, recruited in UKPDS trial centres. In fact, evidence on the presence of circulating autoantibodies in non-insulin-dependent diabetes mellitus in adults has gradually emerged since the late 1970s [13, 14]. In phenotypic T2DM patients older than 65 years, anti-GAD and/or anti-insulinoma-associated protein 2 (IA-2) autoantibodies have been detected in $12 \%$ of patients [15] and the existence of subsets of patients with T2DM phenotype and islet cell autoantibodies has been shown by several studies. To date, the largest cohort examined for diabetes-associated autoantibodies included over 6,000 consecutive adult-onset diabetic patients and revealed that the prevalence of adult-onset autoimmune diabetes in Europe was 9.7\%. GAD autoantibodies were detected in most of these cases, with only a small subset of patients presenting other autoantibodies [16]. Patients aged 30-70 years were designated with LADA if they were autoantibody-positive and did not require insulin treatment for $>6$ months after diagnosis. Overall, LADA was more prevalent than adult-onset T1DM, i.e. autoantibody-positive patients who required insulin treatment $<6$ months after diagnosis, with an OR of 3.3 [16]. LADA is a distinct clinical entity, encompassing phenotypic features of T2DM and immunological similarities to T2DM, which have been extensively reviewed elsewhere [17]. Patients with LADA are heterogeneous, yet they tend to have distinct clinical and pheno- 
Table 1. Clinical characteristics of T2DM patients positive for GAD autoantibodies compared to T2DM patients without autoantibodies

\begin{tabular}{ll}
\hline Characteristic & References \\
\hline Younger age of T2DM onset (<50 years) & $5,16,58-60$ \\
Predominantly female & 16,61 \\
Personal or familial history of autoimmune disease & 5 \\
Higher prevalence of HLA DR3, DQ2, DQ 8 DRB1, DQB1 & $6,19,58,59$ \\
Lower body mass index (less frequently obese) & $5,16,19,59,61-63$ \\
Lower waist-to-hip ratio & $6,16,61$ \\
Lower prevalence of concomitant metabolic syndrome & 62 \\
Lower systolic and/or diastolic blood pressure & 6,16 \\
More likely present with acute symptoms at diagnosis & 5 \\
Higher HbA 1 concentration & 61 \\
Lower fasting plasma insulin levels & 60 \\
Lower fasting C-peptide levels & $6,19,58,63$ \\
Impaired acute phase insulin secretion, lower insulin response to oral glucose load & 6 \\
Less insulin-resistant & 60 \\
Shorter period between diagnosis and initiation of insulin therapy & $16,19,63$ \\
Elevated white blood cell counts, acute phase markers & 36,58 \\
Lower circulating triglyceride concentration & 6,16 \\
Higher high-density lipoprotein cholesterol concentration & 16,62 \\
Increased risk for thyroid autoimmunity and hypothyroidism & 19,62 \\
Less frequent cerebrovascular events & 62 \\
\hline
\end{tabular}

typic characteristics, compared to T2DM patients without signs of autoimmunity: they are primarily younger, more often present with acute symptoms though without ketoacidosis, are less often obese and have a personal or familial history of autoimmune disease [5]. Unsurprisingly, the genetic background of patients with LADA shares features of both T1DM and T2DM [18]. Further clinical features reported for patients with LADA in the literature are presented in table 1.

The phenotypic distinction between patients with low titers of GAD autoantibodies compared with high titers is even more pronounced [19]. High titres of anti-GAD autoantibodies $(>10,000 \mathrm{U} / \mathrm{ml})$ are not necessarily predictive for later insulin deficiency, but are often found in patients with specific HLA DR-DQ genotypes [6]. Interestingly, LADA patients present more frequently with anti-GAD and less often with islet cell antibodies (ICA) or with both types of autoantibodies concomitantly, while antibody clustering consisting of more than one autoantibody is characteristic for T1DM [17]. Longitudinal studies show that GAD autoantibodies and IA-2 autoantibodies remain positive for up to 12 years, although IA-2 autoantibodies decrease more rapidly with disease progression, while GAD autoantibodies tend to remain high even when C-peptide secretion becomes undetectable [20].

Autoimmune Aspects of Type 2 Diabetes Mellitus
Autoantibodies against $\beta$ cells imply ongoing immune-mediated $\beta$-cell destruction, therefore these patients require insulin therapy earlier during disease progression compared to autoantibody-negative patients with T2DM [21]. Specifically, obese and insulin-resistant patients with LADA would require insulin earlier, as their reduced endogenous insulin production will be rapidly insufficient to meet the high insulin requirement. Consequently, the prevention of $\beta$-cell destruction is imperative for these patients.

\section{Autoimmune Activation in T2DM}

Depending on the immunological model one adheres to, autoimmunity occurs due to defects in self-tolerance or the persistence of danger signals stemming from chronic inflammation and tissue destruction $[1,2]$. The latter model, elaborated by Matzinger [2], would favour the concept of autoimmune activation in T2DM, as plenty of pathological alterations characteristic of this disease such as obesity-associated chronic adipose tissue inflammation and $\beta$-cell stress induced by gluco- and lipotoxicity - continuously provide danger signals which cause an activation of both innate and adaptive immunity. Danger signals can include any molecules resulting from cellular 


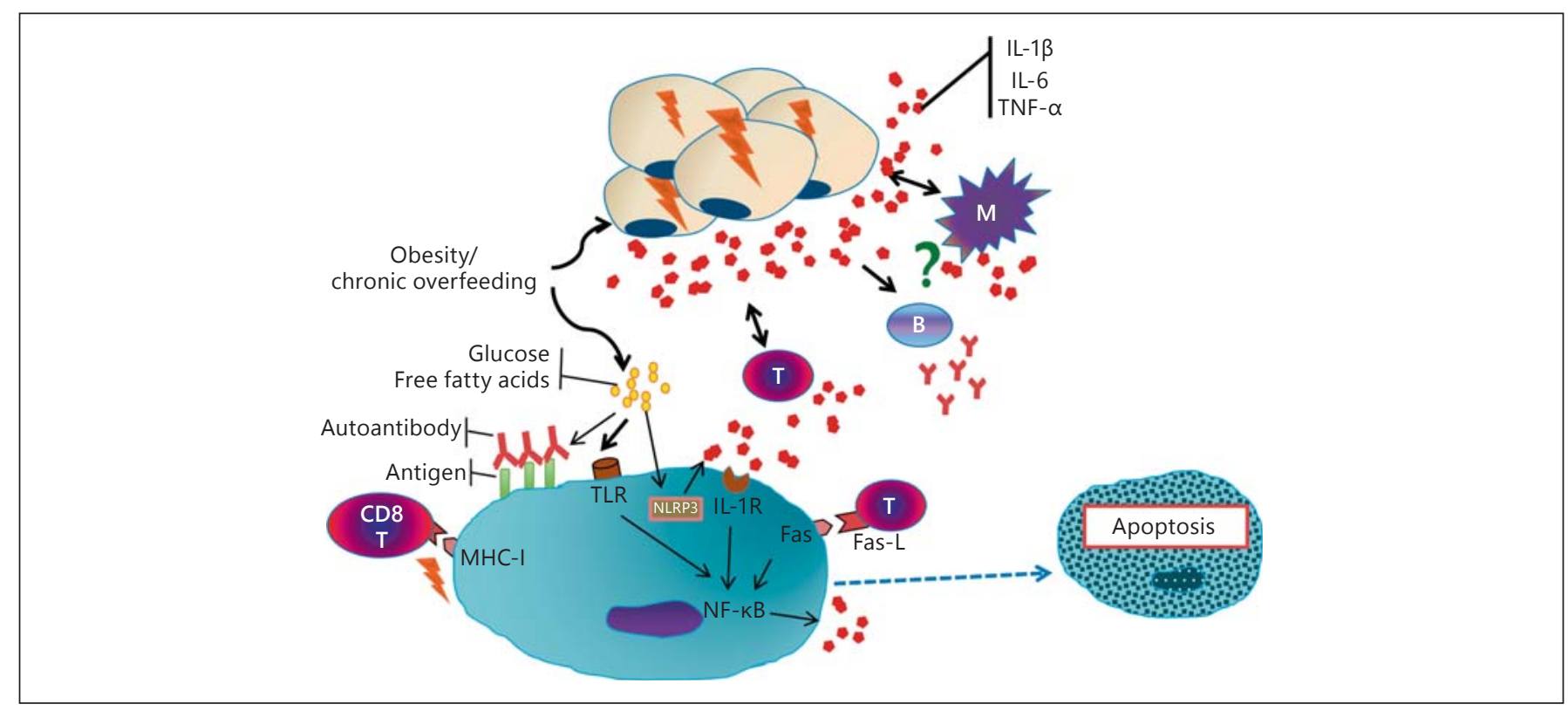

Fig. 1. Islet cell autoimmunity in T2DM. Obesity and chronic overfeeding lead to metabolic and adipocyte stress. Stressed adipocytes secrete pro-inflammatory cytokines such as IL- $1 \beta$, IL- 6 or TNF- $\alpha$, which activate T cells, B cells and macrophages $(M)$. Overfeeding and specifically increased circulating glucose and free fatty acid concentration activates inflammatory signalling via upregulation of the NLRP3 inflammasome and activation TLRs, which leads to increased IL-1 $\beta$ production and further recruitment of macrophages. Hyperglycaemia contributes to an increased expression of several $\beta$-cell antigens, such as GAD, thus increasing the vulnerability of $\beta$ cells to autoantibodies such as anti-GAD, the most frequently detected autoantibodies in phenotypic T2DM. CD8+ T cells may act cytotoxic upon binding to MHC-I molecules on the

distress which binds to pattern recognition receptors, such as toll-like receptors (TLRs) and nucleotide-binding oligomerization domain (NOD) receptors and cause a local or generalized immune response [2]. This response consists in the assembly of a cytosolic protein complex composed of nucleotide-binding and leuchine-rich repeat containing proteins (NLRs) and caspase 1 which, once activated, triggers IL- $1 \beta$ production [22].

Increased circulating glucose concentrations activate the NLRP3 inflammasome while increased free fatty acid concentration activate TLR2 and TLR4, which leads to recruitment of macrophages and eventually $\beta$-cell stress [7]. Figure 1 summarizes our view on $\beta$-cell alterations due to autoimmune and inflammatory mechanisms. 'Glucolipotoxicity'-induced $\beta$-cell apoptosis could favour the release of 'danger signals', autoantibody production, and activation of T cells reactive to $\beta$-cell antigens, culmi- surface of pancreatic $\beta$ cells. Both T cells and macrophages contribute to the amplification of islet inflammation by further releasing inflammatory cytokines, with autocrine and paracrine effects. IL- $1 \beta$ binds to its receptor IL-1R and activates NF- $\mathrm{KB}$. In the presence of IL- $1 \beta, \beta$ cells upregulate Fas expression and become more vulnerable to apoptosis induced by activated $\mathrm{T}$ cells via death domain signalling. Inflammatory alterations targeting the $\beta$ cell can cause the exposure of cryptic antigens which are then recognized by antigen-presenting cells (APC). However, the exact involvement of APC in autoimmune reactions in T2DM is unknown. In the end, the result of all inflammatory and autoimmune reactions is $\beta$-cell apoptosis, further reduction of insulin concentration, and worsening of hyperglycaemia. nating in further autoimmune destruction of $\beta$ cells. Indeed, the expression of several $\beta$-cell antigens is increased when $\beta$ cells are stimulated by glucose and decreased when the $\beta$ cells are less active [23]. Hyperglycaemia increases the expression of GAD in cultured pancreatic rat islets [24]. Binding of ICA from sera to rat pancreas has been shown to be dependent on the metabolic state, feeding rats a high-sucrose and high-fat diet with insulin releasing effects that significantly increase the binding and the endpoint titre of strongly ICA-positive sera, compared to fasting [23]. Following human and rat $\beta$-cell treatment with elevated glucose and free fatty acids levels, $\beta$ cells undergo apoptosis [25]. From the perspective of the 'self/non-self' model, the release of 'cryptic' $\beta$-cell antigens through $\beta$-cell destruction can have immunogenic potential too: if self-reactive T cells, which have escaped deletion in the thymus, encounter such a $\beta$-cell antigen 
presented in primary lymph nodes by dendritic cells, Tcell priming might occur, followed by further T-cell-mediated $\beta$-cell destruction [26]. Stress antigens might drive inflammation in $\beta$ cells, but also in other metabolically affected tissues in T2DM, such as adipose tissue or the vessel wall [27].

The vast majority of T2DM patients are obese and the risk of T2DM increases with the BMI [28]. Wilkin [29] first introduced the hypothesis that obesity and weight gain are crucial for inducing $\beta$-cell apoptosis. According to the 'accelerator hypothesis', weight gain represents an overlay between T1DM and T2DM. Patients who gain a substantial amount of weight become more insulin-resistant and their hyperglycaemia worsens. Hyperglycaemia further contributes to immunogenic destruction of $\beta$ cells due to glucotoxicity, as discussed above [29]. Thus, according to the accelerator hypothesis, autoimmune-mediated $\beta$-cell destruction occurs in both T1DM and T2DM, but the tempo at which $\beta$-cell destruction occurs is significantly lower in T2DM [29].

Although obesity is not a classic risk factor for autoimmune diseases, altered adipokine and cytokine levels detected in obesity as a consequence of chronic adipose tissue inflammation [30] are also reported in some autoimmune conditions [31]. Local and systemic inflammation in obesity and other metabolic disorders has been extensively reviewed elsewhere [32]. Stressed adipocytes secrete pro-inflammatory adipokines and cytokines, which attract $B$ cells, T cells and macrophages. Both T cells and activated macrophages secrete pro-inflammatory cytokines and chemokines, contributing to the persistence of inflammatory reactions within the tissue and subsequently the underlying potential for autoimmune-mediated $\beta$-cell destruction. As presented in figure 1, islets exposed to IL- $1 \beta$ rapidly upregulate Fas expression, making them more susceptible to Fas-mediated NF- $\kappa \mathrm{B}$-induced apoptosis, via interaction with Fas ligand, which is constitutively expressed by many immune cells, including activated T cells $[9,33]$. Interestingly, IL- $1 \beta$ and its antagonist IL-1R levels circulate at increased concentrations in serum of T2DM patients and patients with autoimmune disease and are also predictive for the risk to develop overt diabetes [34]. In addition to harming the $\beta$ cell, pro-inflammatory cytokines promote the recruitment and activation of self-reactive T cells with direct cytotoxic effects [27]. However, altered cytokine and adipokine levels do not always match up in diabetes and autoimmune disease: adiponectin for instance circulates at lower concentration in obese and T2DM patients and negatively correlates with inflammatory markers, while its concentration is in-

Autoimmune Aspects of Type 2 Diabetes Mellitus creased in rheumatoid arthritis or inflammatory bowel disease and positively correlates with inflammatory markers [35]. This seeming inconsistency points to the fact that inflammation accompanying metabolic dysregulation has on the one hand immunogenic potential, but does not overlap completely with autoimmunity on the other.

\section{Lymphocyte Function and Dysfunction in T2DM}

Elevated white blood cell counts are associated with a risk for developing T2DM (OR 1.5 (1.3-1.8)) and autoimmunity may partly explain this association [36]. So far, the role of monocytes and adipose tissue macrophages in T2DM and obesity-associated inflammation has been thoroughly investigated [37], but few studies have addressed the role of $\mathrm{B}$ and $\mathrm{T}$ cells and their products in T2DM or obesity.

Of note, B cells seem to be the first cells to infiltrate adipose tissue upon diet-induced obesity, even before T cells and macrophages at least in mice [38]. NewZealand obese mice are a polygenic model of obesity and T2DM. By disrupting the immunoglobulin heavy chain gene in these mice, they become B-cell-deficient and do not develop diabetes, which emphasizes a role of $B$ cells and autoimmunity in the pathogenesis of T2DM [39]. The pancreas of these mice presents CD19+ B-cell-enriched immune cell aggregates and plasma cells in peri-insular leukocytic infiltrates. A B-cell subset associated with the generation of autoantibodies was also detected in these animals [39]. As previously mentioned, evidence of lymphocytic infiltrates in target organs such as the pancreas, underlines the idea of autoimmune participation in T2DM pathogenesis.

When compared to B cells of healthy subjects, B cells of patients with T2DM fail to secrete the anti-inflammatory interleukin-10 (IL-10) in response to stimulation through TLR2, TLR4 or TLR9 [40]. IL-10 secreted by B cells is important for controlling autoimmune processes [41]. Accordingly, low IL-10 production in response to stimulation with lipopolysaccharide was associated with a high risk for T2DM in elderly subjects [42]. It is thus conceivable that dysfunctional B cells might add to altered IL-10 production observed in T2DM.

$\mathrm{T}$ cells are more established players intertwined in metabolic disorders and represent a strong link between inflammatory and autoimmune alterations. BrooksWorrell et al. [43] demonstrated that islet-reactive T cells can be found in phenotypic T2DM patients and the presence of such cells is associated with a more severe $\beta$-cell 
lesion and lower residual insulin secretion. They established an assay to measure T-cell reactivity to islet antigens and discovered the existence of a T-cell population responsive to several islet antigens in LADA patients but also phenotypic T2DM patients without autoantibodies [43]. This crucial finding is another important link between autoimmunity and T2DM.

Besides self-reactive T cells, obesity is associated with an imbalance in Th1/Th2 ratio, which leads to the secretion of more pro-inflammatory adipokines, cytokines and chemokines which help attract more immune cells in the pancreas and adipose tissue of obese T2DM patients [11]. Another T-cell subset, Th17, participates in the induction of autoimmunity and inflammation and their abundance is enhanced in obesity [44]. The increased number of Th17 and CD8+ T cells in T2DM and obesity may also link inflammation to islet autoimmune destruction. Tregs are necessary for maintaining self-tolerance and immune regulation and include several subsets [45]. They exert their suppressive function through direct cellcell contact and production of anti-inflammatory cytokines such as IL-10. In mouse models of obesity and T2DM, the number of Tregs in visceral adipose tissue is drastically reduced [46], whereas induction of Tregs reduces inflammation and mitigates autoimmune reactions, insulin resistance and end-organ complications [10]. However, our group could not find evidence for reduced Tregs in human obesity, but we could show that inflammation correlates with markers of T-cell subsets including Tregs in adipose tissue from obese patients [47]. Nevertheless, the relevance of Tregs in the pathogenesis of T2DM in humans is still poorly understood.

\section{Immunomodulatory Treatment}

Despite unequivocally being an autoimmune disease, antigen-specific therapies have not yet found the way into clinic routine for the treatment of T1DM. Considering the fact that autoimmune reactions contribute to the pathogenesis of T2DM as well, but to a limited extent, antigen-specific therapies are still not in sight for prevention or treatment of T2DM. However, keeping in mind the prominent role of innate immune system activation in adipose tissue inflammation and T2DM, anti-inflammatory and immunomodulatory therapeutic approaches may be beneficial in improving metabolic regulation in T2DM.

Promising results have initially been achieved by targeting IL- $1 \beta$, an inflammatory cytokine with a pivotal role in islet inflammation in T2DM [7]. Antagonizing its effects by IL-1R antagonist, anakinra, proved favourable for improvement of glucose control in T2DM patients randomized to receive either the drug or placebo for 13 weeks [48]. At the end of the treatment, a significant reduction in $\mathrm{HbA}_{1 \mathrm{c}}$ levels occurred, parallel to an increase in circulating $\mathrm{C}$-peptide concentration and proinsulin, all pointing towards an improved $\beta$-cell function. During the follow-up period, which lasted 39 weeks and was intervention-free, patients showed a pronounced decrease in C-peptide levels and a lower ratio of proinsulin to insulin [48].

One case report is definitely worth mentioning in this context. Ursini et al. [49] showed a dramatic improvement of insulin sensitivity after 12 weeks of treatment with CTLA4 immunoglobulin (abatecept) in a severely insulin-resistant patient suffering from rheumatoid arthritis. The improvement in insulin sensitivity was not accompanied by a reduction of systemic inflammation, thus the authors assumed that the insulin-sensitising effect of the drug is solely attributable to T-cell suppression.

High doses of salicylates block the NF- $\kappa$ B pathway and increase circulating adiponectin, an important adipokine counteracting insulin resistance. In a randomized clinical trial, a high dose of salsalate improved metabolic control and $\mathrm{HbA}_{1 \mathrm{c}}$ levels in T2DM patients [50]. Salsalate is a prodrug of salicylate with reduced undesirable bleeding and gastrointestinal effects emphasizing its use as an insulin sensitizer. However, recent data suggest that part of the glucose-lowering action of salsalate is due to activation of AMP kinase [51].

Long-chain $n-3$ polyunsaturated fatty acids (PUFA) are known for their anti-inflammatory actions and immunomodulating effects on T cells [52]. We have recently shown in a randomized controlled trial that treatment of obese, mostly insulin-resistant but non-diabetic patients that the resolving lipid mediator precursors eicosapentaenoic acid (EPA) and docosahexaenoidic acid (DHA) lead to a reduced expression of key inflammatory genes adipose tissue, lower circulating IL-6 concentration parallel to a substantial increase in anti-inflammatory resolving lipid mediators resolvins $\mathrm{D}_{1}$ and $\mathrm{E}_{1}$ as well as protectin $\mathrm{D}_{1}[53]$.

TLRs can be inappropriately activated by self-components and cause inflammation and autoimmunity and have been hypothesized to play a role in the pathogenesis of T2DM. TLRs are downregulated by angiotensin receptor blockers and these substances protected pancreatic islets and reduced adipose tissue inflammation in mice fed a high-fat diet [54]. 
Dipeptidyl peptidase (DPP) inhibitors and GLP-1 analogues alter the inflammatory profile and reduce inflammatory cytokine secretion, while improving glucose metabolism [55]. Interestingly, the DPP-4 inhibitor sitagliplin reduces autoimmunity by decreasing the homing of $\mathrm{CD} 4+$ cells into pancreatic $\beta$ cells in NOD mice and helps preserve islet cell mass [56].

Immunological tolerance may be induced by oral antiCD3 therapies, which may be clinically applicable for chronic treatment due to the reduced side effects, including cytokine release syndrome [57]. Since antigens in T2DM are not well defined, induction of antigen nonspecific Tregs by oral anti-CD3 is preferable to parenteral administration and will be the focus of intense research in the future.

\section{Future Outlook}

Autoimmunity may be both cause and consequence of $\beta$-cell dysfunction, imposing in either case a further disturbance in glucose homeostasis. The prevalence of $\mathrm{T} 2 \mathrm{DM}$ is on the rise and if $10 \%$ of these patients are positive for islet autoantibodies, then testing for islet autoantibodies as part of the diagnostic assessment in T2DM is relevant to a great number of adult patients, as it may contribute to the rate of progression to insulin requirement, particularly in the absence of gross visceral obesity. Autoantibodies indeed help distinguish adult patients with T1DM, LADA or T2DM, but also the presence of self-reactive $T$ cells in autoantibody-negative T2DM patients identifies an autoimmunity that is associated with the metabolic dysregulation. Finding a cause of autoimmune activation in T2DM in future research is surely an interesting path worth pursuing, as the immunogenic basis of T2DM may not be restricted to chronic inflammation in many patients. The identification of autoimmune aspects in patients with T2DM also relies on successful metabolic control by immunomodulatory treatment that is still to be shown in the long term. Then the proper T2DM subclassification has essential therapeutic implications as immunomodulatory regimens might efficiently prevent or slow down autoimmune destruction of $\beta$ cells. Surely sustained future research is needed in order to understand the complex immune interactions relevant to T2DM, but we should remember that basic measures in the prevention and management of T2DM such as lifestyle changes also repress inflammation and autoimmune reactions, and are therefore a major tool to help decrease $\beta$-cell stress and compensate for the relative lack of insulin.

\section{Acknowledgements}

This work was supported by the Federal Ministry of Economy, Family and Youth and the National Foundation for Research, Technology and Development (all to T.M.S.). We thank Maximilian Zeyda and Alexander Jürets for helpful discussions. We apologize to many authors whose work we were unable to cite due to lack of space.

\section{Conflict of Interest}

The authors have no conflicts of interest to disclose.

\section{References}

1 Kamradt T, Mitchison NA: Tolerance and autoimmunity. N Engl J Med 2001;344:655664.

2 Matzinger P: The danger model: a renewed sense of self. Science 2002;296:301-305.

-3 Mackay IR: Clustering and commonalities among autoimmune diseases. J Autoimmun 2009;33:170-177.

4 Wentworth JM, Fourlanos S, Harrison LC: Reappraising the stereotypes of diabetes in the modern diabetogenic environment. Nat Rev Endocrinol 2009;5:483-489.

5 Fourlanos S, et al: A clinical screening tool identifies autoimmune diabetes in adults. Diabetes Care 2006;29:970-975.

-6 Tuomi T, et al: Clinical and genetic characteristics of type 2 diabetes with and without GAD antibodies. Diabetes 1999;48:150-157.
7 Donath MY, Shoelson SE: Type 2 diabetes as an inflammatory disease. Nat Rev Immunol 2011;11:98-107.

8 Gallucci S, Matzinger P: Danger signals: SOS to the immune system. Curr Opin Immunol 2001;13:114-119.

-9 Maedler K, et al: Glucose induces $\beta$-cell apoptosis via upregulation of the Fas receptor in human islets. Diabetes 2001;50:1683-1690.

10 Eller K, et al: Potential role of regulatory T cells in reversing obesity-linked insulin resistance and diabetic nephropathy. Diabetes 2011;60:2954-2962.

11 Poggi M, et al: The inflammatory receptor CD40 is expressed on human adipocytes: contribution to crosstalk between lymphocytes and adipocytes. Diabetologia 2009;52:11521163 .
12 Turner R, et al: UKPDS 25: autoantibodies to islet-cell cytoplasm and glutamic acid decarboxylase for prediction of insulin requirement in type 2 diabetes. UK Prospective Diabetes Study Group. Lancet 1997;350:12881293.

13 Irvine WJ, et al: Clinical and pathogenic significance of pancreatic-islet-cell antibodies in diabetics treated with oral hypoglycaemic agents. Lancet 1977;1:1025-1027.

-14 Tuomi T, et al: Antibodies to glutamic acid decarboxylase reveal latent autoimmune diabetes mellitus in adults with a non-insulindependent onset of disease. Diabetes 1993;42: 359-362.

15 Pietropaolo M, et al: Evidence of islet cell autoimmunity in elderly patients with type 2 diabetes. Diabetes 2000;49:32-38. 
16 Hawa MI, et al: Adult-onset autoimmune diabetes in Europe is prevalent with a broad clinical phenotype: action LADA 7. Diabetes Care 2013;36:908-913.

17 Naik RG, Brooks-Worrell BM, Palmer JP: Latent autoimmune diabetes in adults. J Clin Endocrinol Metab 2009;94:4635-4644.

18 Cervin C, et al: Genetic similarities between latent autoimmune diabetes in adults, type 1 diabetes, and type 2 diabetes. Diabetes 2008; 57:1433-1437.

19 Falorni A, et al: Autoantibody recognition of $\mathrm{COOH}$-terminal epitopes of GAD65 marks the risk for insulin requirement in adult-onset diabetes mellitus. I Clin Endocrinol Metab 2000;85:309-316.

20 Borg H, et al: A 12-year prospective study of the relationship between islet antibodies and $\beta$-cell function at and after the diagnosis in patients with adult-onset diabetes. Diabetes 2002;51:1754-1762.

21 Pozzilli P, Di Mario U: Autoimmune diabetes not requiring insulin at diagnosis (latent autoimmune diabetes of the adult): definition, characterization, and potential prevention. Diabetes Care 2001;24:1460-1467.

22 Medzhitov R: Origin and physiological roles of inflammation. Nature 2008;454:428-435.

23 McCulloch DK, Barmeier H, Neifing JL, Palmer JP: Metabolic state of the pancreas affects end-point titre in the islet cell antibody assay. Diabetologia 1991;34:622-625.

24 Hao W, et al: Functional state of the $\beta$ cell affects expression of both forms of glutamic acid decarboxylase. Pancreas 1994;9:558-562.

25 Lupi R, et al: Prolonged exposure to free fatty acids has cytostatic and pro-apoptotic effects on human pancreatic islets: evidence that $\beta$-cell death is caspase mediated, partially dependent on ceramide pathway, and Bcl-2 regulated. Diabetes 2002;51:1437-1442.

-26 Brooks-Worrell B, Palmer JP: Immunology in the Clinic Review Series; focus on metabolic diseases: development of islet autoimmune disease in type 2 diabetes patients: potential sequelae of chronic inflammation. Clin Exp Immunol 2012;167:40-46.

-27 Lukens JR, Dixit VD, Kanneganti TD: Inflammasome activation in obesity-related inflammatory diseases and autoimmunity. Discov Med 2011;12:65-74.

28 Wild S, et al: Global prevalence of diabetes: estimates for the year 2000 and projections for 2030. Diabetes Care 2004;27:1047-1053

29 Wilkin TJ: The accelerator hypothesis: weight gain as the missing link between type 1 and type 2 diabetes. Diabetologia 2001;44:914922.

30 Trayhurn P, Wood IS: Adipokines: inflammation and the pleiotropic role of white adipose tissue. Br J Nutr 2004;92:347-355.

-31 Fantuzzi G: Adipose tissue, adipokines, and inflammation. J Allergy Clin Immunol 2005; 115:911-919; quiz 920.
32 Zeyda M, Stulnig TM: Obesity, inflammation, and insulin resistance - a mini-review. Gerontology 2009;55:379-386.

33 Maedler K, et al: Glucose-induced $\beta$-cell production of IL- $1 \beta$ contributes to glucotoxicity in human pancreatic islets. J Clin Invest 2002; 110:851-860.

34 Meier CA, et al: IL-1 receptor antagonist serum levels are increased in human obesity: a possible link to the resistance to leptin? J Clin Endocrinol Metab 2002;87:1184-1188.

35 Fantuzzi G: Adiponectin and inflammation: consensus and controversy. J Allergy Clin Immunol 2008;121:326-330.

36 Schmidt MI, et al: Markers of inflammation and prediction of diabetes mellitus in adults (Atherosclerosis Risk in Communities Study): a cohort study. Lancet 1999;353:1649-1652.

$37 \mathrm{Xu} \mathrm{H}$, et al: Chronic inflammation in fat plays a crucial role in the development of obesityrelated insulin resistance. J Clin Invest 2003; 112:1821-1830

38 Duffaut C, et al: Unexpected trafficking of immune cells within the adipose tissue during the onset of obesity. Biochem Biophys Res Commun 2009;384:482-485.

39 Haskell BD, Flurkey K, Duffy TM, Sargent EE, Leiter EH: The diabetes-prone NZO/HILt strain. I. Immunophenotypic comparison to the related NZB/BlNJ and NZW/LacJ strains. Lab Invest 2002;82:833-842.

40 Jagannathan M, et al: Toll-like receptors regulate B-cell cytokine production in patients with diabetes. Diabetologia 2010;53:14611471.

41 Fillatreau S, et al: B cells regulate autoimmunity by provision of IL-10. Nat Immunol 2002;3:944-950.

42 Van Exel E, et al: Low production capacity of interleukin-10 associates with the metabolic syndrome and type 2 diabetes: the Leiden $85-$ Plus Study. Diabetes 2002;51:1088-1092.

43 Brooks-Worrell BM, et al: Identification of autoantibody-negative autoimmune type 2 diabetic patients. Diabetes Care 2011;34:168173.

44 Winer S, et al: Obesity predisposes to Th1 bias. Eur J Immunol 2009;39:2629-2635.

45 Shevach EM: From vanilla to 28 flavors: multiple varieties of T-regulatory cells. Immunity 2006;25:195-201.

46 Feuerer M, et al: Lean, but not obese, fat is enriched for a unique population of regulatory $\mathrm{T}$ cells that affect metabolic parameters. Nat Med 2009;15:930-939.

47 Zeyda M, Huber J, Prager G, Stulnig TM: Inflammation correlates with markers of T-cell subsets including regulatory $\mathrm{T}$ cells in adipose tissue from obese patients. Obesity (Silver Spring) 2011;19:743-748.

48 Larsen CM, et al: Sustained effects of interleukin-1 receptor antagonist treatment in type 2 diabetes. Diabetes Care 2009;32:1663-1668.
49 Ursini F, et al: Improvement in insulin resistance after short-term treatment with abatacept: case report and short review. Clin Rheumatol 2012;31:1401-1402.

50 Goldfine $\mathrm{AB}$, et al: The effects of salsalate on glycemic control in patients with type 2 diabetes: a randomized trial. Ann Intern Med 2010;152:346-357.

51 Hawley SA, et al: The ancient drug salicylate directly activates AMP-activated protein kinase. Science 2012;336:918-922.

52 Zeyda M, et al: Suppression of T-cell signaling by polyunsaturated fatty acids: selectivity in inhibition of mitogen-activated protein kinase and nuclear factor activation. J Immunol 2003;170:6033-6039.

53 Itariu BK, et al: Long-chain n-3 PUFAs reduce adipose tissue and systemic inflammation in severely obese nondiabetic patients: a randomized controlled trial. Am J Clin Nutr 2012;96:1137-1149.

54 Cole BK, et al: Valsartan protects pancreatic islets and adipose tissue from the inflammatory and metabolic consequences of a high-fat diet in mice. Hypertension 2010;55:715-721.

55 Duez H, Cariou B, Staels B: DPP-4 inhibitors in the treatment of type 2 diabetes. Biochem Pharmacol 2012;83:823-832.

56 Kim SJ, Nian C, Doudet DJ, McIntosh CH: Dipeptidyl peptidase IV inhibition with MK0431 improves islet graft survival in diabetic NOD mice partially via T-cell modulation. Diabetes 2009;58:641-651.

57 Da Cunha AP, Weiner HL: Induction of immunological tolerance by oral anti-CD3. Clin Dev Immunol 2012;2012:425021.

58 Bruno G, et al: Clinical, immunological, and genetic heterogeneity of diabetes in an Italian population-based cohort of lean newly diagnosed patients aged 30-54 years. Piedmont Study Group for Diabetes Epidemiology. Diabetes Care 1999;22:50-55.

59 Kelly MA, et al: HLA typing and immunological characterization of young-onset diabetes mellitus in a Hong Kong Chinese population. Diabet Med 2001;18:22-28.

60 Unnikrishnan AG, Singh SK, Sanjeevi CB Prevalence of GAD65 antibodies in lean subjects with type 2 diabetes. Ann NY Acad Sci 2004;1037:118-121.

61 Genovese S, et al: Clinical phenotype and $\beta$-cell autoimmunity in Italian patients with adult-onset diabetes. Eur J Endocrinol 2006; 154:441-447.

62 Romkens TE, Kusters GC, Netea MG, Netten PM: Prevalence and clinical characteristics of insulin-treated, anti-GAD-positive, type $2 \mathrm{di}$ abetic subjects in an outpatient clinical department of a Dutch teaching hospital. Neth J Med 2006;64:114-118.

63 Gambelunghe G, et al: Increased risk for endocrine autoimmunity in Italian type 2 diabetic patients with GAD65 autoantibodies. Clin Endocrinol (Oxf) 2000;52:565-573. 